\title{
Factors Affecting Operation of Apparatus for Counting Alpha Particles in an Ion Counting Chamber
}

\author{
By F. J. Davis
}

\begin{abstract}
The resolving times of two amplifier circuits for the counting of alpha particles in a cylindrical ion chamber is discussed. The original circuit, using a floating grid on the first stage of the amplifier, gave a resolving time of $2.5 \times 10^{-3}$ second. An improved circuit using a 1-megohm grid resistor on the first stage lowered the resolving time to $1.4 \times 10^{-4}$ second

The effect on the counting rate of the presence of small amounts of $\mathrm{CO}, \mathrm{CO}_{2}, \mathrm{H}_{2} \mathrm{O}$ and $\mathrm{HCl}$ in the ion chamber was found to be negligible. The presence of 1 percent of air. however, reduces the counting efficiency by 20 percent.

A method whereby the collection time of radon in radium solutions may be of the order of $1 / 2$ hour is given. Experiments show that boiling and bubbling nitrogen through 200 ml radium solutions for 17 minutes removes 99.99 percent of the radon. The absorption of radon by small neoprene meteorlogical balloons was found to be approximatly $(0.03 \%$ l $\min ) / 100 \mathrm{~cm}^{2}$.
\end{abstract}

\section{Introduction}

In the operation of the alpha particle counting apparatus for the determination of radium by the radon method ${ }^{1}$ swo problems that have been investigated are (1) the resolution of the electronic pulses resulting from the alpha particles and (2) the quantitative transfer of the radon representing the radium.

Factors contributing to the first problem are the resolving time of the electronic circuit and the composition of the gas. Pulses occuring within an interval less than the resolving time are recorded as a single pulse. This affects the calibration constant so that it is a function of counting rate as well as resolving time. The electronic pulses are essentially produced by the collection of electrons on the center electrode of the ion chamber. In the presence of gases such as oxygen, which have a high affinity for electrons, the electrons are captured with the formation of heavy negative ions of low mobility. As a result, the pulses are broadened and reduced in height so that many pulses fail to reach the threshold pulse

${ }^{1}$ L. F. Curtiss and F. J. Davis, J. Research NBS 31, 181 (1943) RP1557. height that will just activate the pulse discriminator and thus fail to be recorded.

Factors involved in the second problem (transfer of radon) are (1) deemanation time, (2) collection time, and (3) absorption of radon. The deemanation time or the time required to extract the radon from the solution should be sufficient to transfer all radon from the solution to the chamber, and it will depend on the volume of the ion chamber as well as on the amount of solution. The collection time, that is, the time allowed for the growth of radon in the radium solution, should be sufficiently well defined so that the amount of radon produced may serve as a measure of the radium present. This is especially critical when this time interval is short. The absorption of radon in rubber is important in the taking of breath samples that are usually obtained by the inflation of a balloon.

\section{Resolving Time}

The resolving time used in this paper is defined by the relation $N=N_{0} e^{-f t}$, where $N$ is the count per hour per $10^{-12}$ curie, $t$ the resolving time, $f$ the 
actual count per hour and $N_{\mathrm{o}}$ is the count per hour per $10^{-12}$ curie for $f=0$.

The calibration constant $N$ becomes smaller the larger the resolving time. The immediate advantage of a short resolving time, seen from the formula, is that larger samples may be measured without having to apply a large correction to allow for unresolved pulses. As the statistical error of a random count is determined by the square root of its total count, a further advantage gained by a higher counting rate is that greater accuracy is obtained in a given time of measurement.

If the apparatus used is an ion chamber with an amplifying device, such as a $\mathrm{FP}-54$ circuit with a galvanometer, the resolving time may be of the order of 1 second, thus limiting the radon from the sample measured to the order of $10^{-12}$ curie. With this limitation, samples that must be fused in a vacuum furnace may require quantities of the order of milligrams or iess, thereby introducing sampling errors.

In figure 1 is shown the calibration or count per hour per $10^{-12}$ curie for different counting

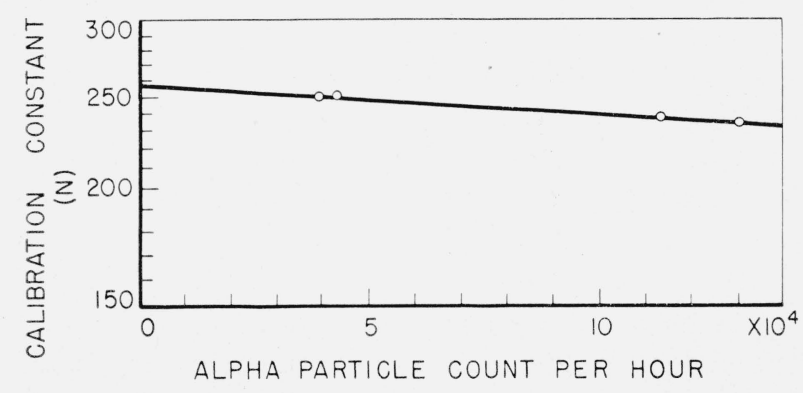

FIGURE 1.-Variation of calibraition count per hour per $10^{-12}$ curie with counting rate for the original circuit.

Resolving time calculated from slope of curve equals $2.5 \times 10^{-3} \mathrm{sec}$.

rates for the apparatus as described in a previous paper. $^{2}$ The count per hour per $10^{-12}$ curie is plotted as the ordinate on a logarithmic scale and the counting rate as the abscissa on a linear scale. It is to be nocied that the calibration constant $N$ drops from 257 counts/hr per $10^{-12}$ curie for small counts, to 239 for a counting rate of 100,000 counts $/ \mathrm{hr}$, which is the equivalent of $4.2 \times 10^{-10}$ curie.

Calculating $t$ from the slope of the line in figure 1 gives $t=2.5 \times 10^{-3} \mathrm{sec}$. Although this resolving time is adequate for breath samples and room air 2 See footnote 1 . samples where the amount of radon rarely exceeds $5 \times 10^{-11}$ curie, it requires the use of the calibration curve for samples over this value.

In the amplifier used to secure the data shown in figure 1, no grid leak was used on the first amplification stage (type 38 tube). To shorten the resolving time for this circuit, the obvious method was the placing of a 1-megohm grid leak between the grid of the 38 tube and ground. The effect of this is shown in figure 2. The

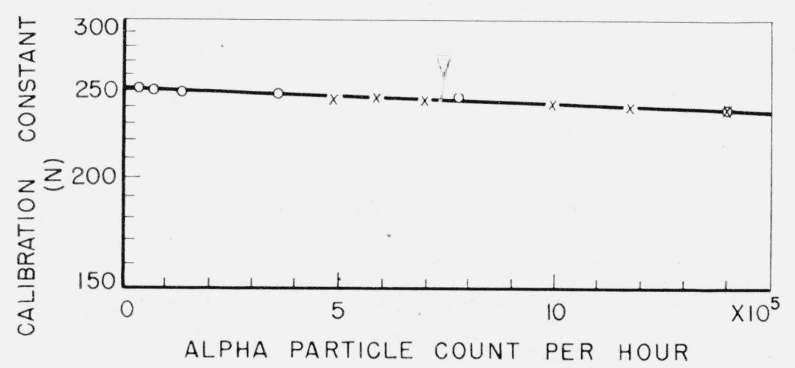

FIG. 2.-Variation of calibraition count per hour per $10^{-12}$ curie with the counting raie for short resolving time circuit.

Resolving time calculated from slope of curve equals $1.4 \times 10^{-4} \mathrm{sec}$. The circles represent independent samples of radon. The crosses represent different recounts of a single sample while decaying during a period of 1 week.

calibration count per hour per $10^{-12}$ curie drops from 252 for small counting rates to 238 for a counting rate of $1,400,000$ counts/hr. It is to be noted that the counting rate in figure 2 covers a tenfold range of that shown in figure 1. Calculating the resolving time from the slope of the line gives $t=1.4 \times 10^{-4}$ sec.

These two curves are for the same chamber, 38 tube and amplifier. For the first curve, the circuit for the 38 tube and amplifier was as suggested by Waddell. $^{3}$ In our amplifier a coupling condenser of $500 \mu \mu \mathrm{f}$ was used for the plate of the 38 tube to the grid of the following stage. For the second curve the changes made in the circuit of the 38 tube were (1) replacing the $500-\mu \mu \mathrm{f}$ coupling condenser on the plate with a $100-\mu \mu \mathrm{f}$ condenser, (2) connecting a 1-megohm grid leak to the ground, (3) limiting the cathode emission by placing a resistor of about $30 \mathrm{ohms}$ in parallel with the heater, and (4) the 38 tube was connected as a triode, that is, with the screen grid and plate connected together.

The original circuit with the longer resolving time has a greater efficiency of counting at slow

${ }^{3}$ R. C. Waddell, Rev. Sci. Instruments 10, 311 (1939). 
rates as shown by comparing figures 1 and 2 . This increased efficiency is probably due to a better signal to noise ratio, which is somewhat higher for the original circuit. As the number of pulses that are below the threshold pulse height in either circuit is small, the difference in the efficiency of counting by the two circuits amounts to only 2 perent.

In figure 2 the calibration curve for the short resolving time circuit shows both circles and crosses. The circles are independent fillings of the chambers from two different standards, one containing $10^{-9} \mathrm{~g}$ Ra for the lower counting rates and the other $1.077 \times 10^{-8} \mathrm{~g}$ Ra for the higher counting rate. The crosses are all from a single filling and represent different recounts, beginning on the right and proceeding to the left, as the radon decayed in the chamber. The value used for $\lambda$, the decay constant of radon was $0.00755 / \mathrm{hr}$. The decay of the radon was followed for 1 week, and each cross was the average of a 12-hour run with 24 hours between the beginning of each run, except between the third and fourth crosses from the right, when it was 48 hours.

A side tube containing water was sealed on a chamber and the temperature of the water controlled. No effect on the counting rate was noted for temperatures below room temperature. At room temperature spurious pulses occurred, probably due to leakage across insulators in the chamber. A single drying trap using $\mathrm{CaCl}_{2}$ removes water vapor sufficiently for satisfactory operation.

Hydrogen chloride of the amount driven off from deemanating a 5-percent $\mathrm{HCl}$ solution has no effect on the counting rate. However, if strong $\mathrm{HCl}$ solutions such as constant boiling mixtures are used, the counting rate is reduced. A trap filled with Ascarite followed by a drying trap can be used to remove the hydrogen chloride.

The $\mathrm{CO}$ and $\mathrm{CO}_{2}$ were taken from commercial gas cylinders and passed through the purification system described in a previous paper ${ }^{4}$ which removed the oxygen and water vapor. In the case of $\mathrm{CO}$, an additional trap of Ascarite plus a drying agent was used to remove any $\mathrm{CO}_{2}$ present.

4 See footnote 1 .

\section{Effect of Composition of Gas}

The effect on the calibration constant of the presence of carbon monoxide, carbon dioxide, air, and water vapor was determined. Considerable quantities of $\mathrm{CO}$ are formed by a carbon-crucible vacuum-furnace when oxygen is liberated from the samples fused. The effect of using $\mathrm{CO}$ in place of nitrogen in the ion chamber was to lower the calibration constant by only 1 percent.

A small amount of $\mathrm{CO}_{2}$ seems to decrease the resolving time of the apparatus. The effect of the addition of $\mathrm{CO}_{2}$ is shown in figure 3. The ordinate represents the resolving time in $10^{-4}$ sec, and the abscissa represents the percent by volume of $\mathrm{CO}_{2}$ in the chamber. The resolving time falls to a minimum near 10 percent of $\mathrm{CO}_{2}$. whereas at 20 percent of $\mathrm{CO}_{2}$ it is about the same as pure nitrogen and at greater percentages the resolving time rises. The effect of $\mathrm{CO}_{2}$ on the calibration constant for concentrations less than 20 percent at a counting rate of 250,000 counts/hr is less than $1 / 2$ percent.

The effect of air in the chambers is very marked; 1 percent of air reduces the counting efficiency at 30,000 counts/hr by approximately 20 percent.

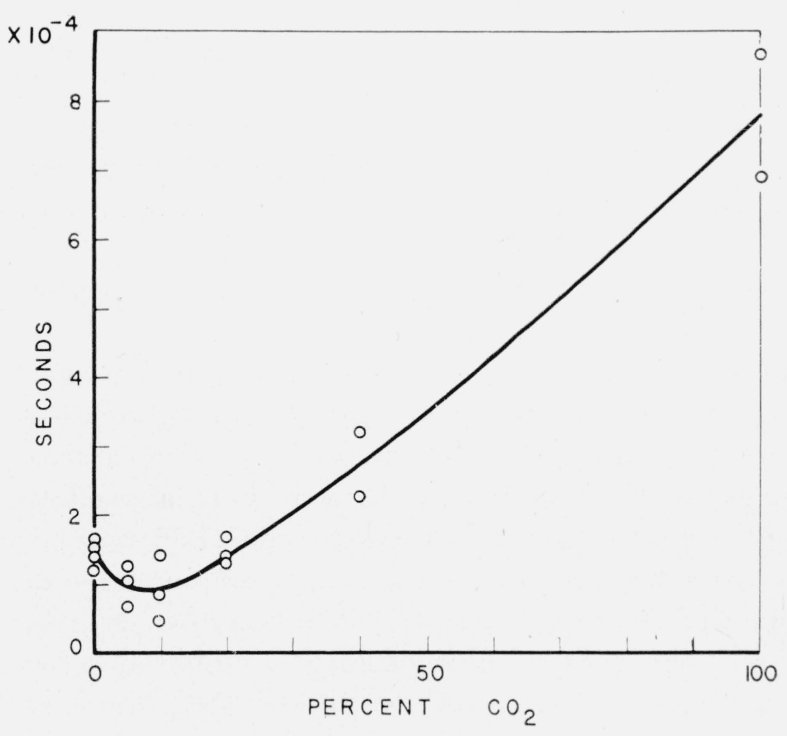

Figure 3.-Curve showing effect of $\mathrm{CO}_{2}$ on resolving time.

The circles represent observations made by using the short resolving time circuit. 


\section{Deemanation Time}

The method used for deemanating solutions is to boil the solutions under reduced pressure of approximately $1 / 2$ atmosphere in a reflux condenser while bubbling $\mathrm{N}_{2}$ through it as the transfer agent to carry the radon into the chamber. As the chamber is filled to atmospheric pressure, the pressure and boiling temperature in the condenser rises accordingly. The time required to fill the chamber is usually 16 to 18 minutes in order to efficiently remove $\mathrm{O}_{2}$.

A test was made to measure the efficiency of transferring the radon from a $200-\mathrm{ml}$ solution for various deemanation times into a 3.6-liter chamber. This was accomplished by filling a chamber in a specified time and immediately on completion reboiling the same solution and filling a second chamber to measure any radon remaining in the solution. For a 17-minute deemanation time, no radon was found in the solution in excess of that amount that would grow from the radium in the solution in the short time between the two runs. The efficiency of the above test was such that one-hundredth of a percent would have been detected. For a 10.5-minute run, $0.020 \pm 0.003$ percent of the radon remained in the solution. For a run of 6 minutes 1.01 percent of the radon remained.

The results of a slightly different experiment to determine the amount of radon transferred during a normal run of 16 minutes is shown in figure 4 , where the amount of radon removed from a solution containing $1.62 \times 10^{-10}$ curie of radon is plotted against the time from the start of the deemanation. In this case, the flow of nitrogen through the solution was adjusted to a rate that would normally fill a chamber to atmospheric pressure in 16 minutes. It was then allowed to flow for the first minute into chamber number one; for the second minute into chamber number two; for the next 2 minutes into chamber number three; the next 4 minutes into chamber number four, and the remaining 8 minutes into chamber number five. After the deemanation was completed, the chambers were filled to atmospheric pressure with $\mathrm{N}_{2}$. The amount of radon transferred at the termination of the filling of any one chamber would then be the sum of the amount of radon in the chamber and the preceding chambers filled.

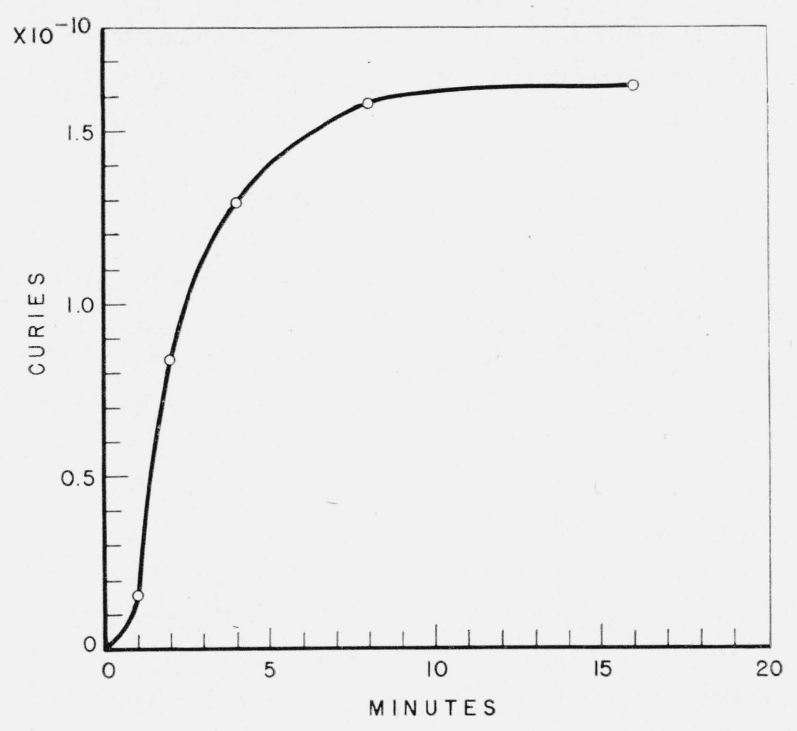

FIGURE 4.-Curve showing radon transferred from reflux condenser to ion chamber during deemanation.

Total amount of radon transferred $1.62 \times 10^{-10}$ curie.

During the first minute of deemanation the stopcocks are being adjusted, consequently the first point may vary considerably for different runs. According to the curve in figure 4 the radon transferred in the first 6 minutes is 92 percent. As only a portion of the nitrogen has passed through the solution to fill a chamber, less radon is transferred than when all the nitrogen passes through the solution to fill a chamber as in the earlier experiment.

\section{Collection Time}

The time allowed for the collection of the radon from a sample is the interval between a preliminary deemanation and the filling of the chamber by a second deemanation process. This interval is measured from the instant when the stopcocks are closed on the reflux condenser at the end of the first deemanation to the exact instant during the subsequent filling of the ion chamber when all the radon destined to enter the chamber has been removed from the solution. The collection time terminates before the ionization chamber stopcock is closed, as it takes some time for the radon to pass through the purification system. The effective point of termination of the collection is fixed according to the following consideration. Suppose first that the ionization chamber is filled to atmospheric pressure and the purifying 
system evacuated. If then the stopcock $T_{3}$ in figure 5 is opened, the mercury manometer will come to a level $B$ depending on the relative volumes of the ionization chamber and the purifying system. Vice versa, in our actual operation, when the manometer reaches the level $B$, we know that all the radon contained in the purifying system and that radon alone will be swept into the ionization chamber when this is filled up to atmospheric pressure. All that is necessary to determine the time of the second deemanation is to record the time when the mercury crosses the mark $B$ while filling the chamber. The radon accumulated in the additional time of filling fails to reach the chamber.

Using this interval for short time collections proved to be accurate within the statistical error of 0.3 percent, even for a collection time of 30 minutes.

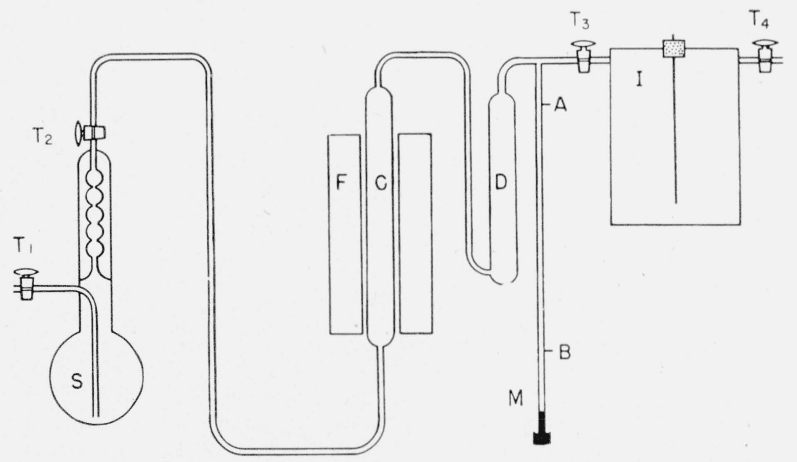

Figure 5.-Diagram of apparatus for filling chambers.

$F$, Furnace; $C$, quartz tube containing reduced copper gauze; $D$, drying tube; $M$, mercury manometer; $I$, ion chamber; $S$, reflux condenser containing radium solution or standard.

\section{Absorption of Radon}

The absorption of radon by small neoprene meteorological balloons, such as are sometimes used in transferring samples, is shown in figure 6 . The percent absorption of radon is plotted against time of contact with the neoprene. The area of the balloon used was about $2,500 \mathrm{~cm}^{2}$. Using this value, the amount of absorption is approximately $(0.03 \% / \mathrm{min}) / 100 \mathrm{~cm}^{2}$. The absorption was measured by placing a known amount of radon in a balloon, allowing it to stand for the period of time under consideration, and then measuring the remaining radon. The amount of

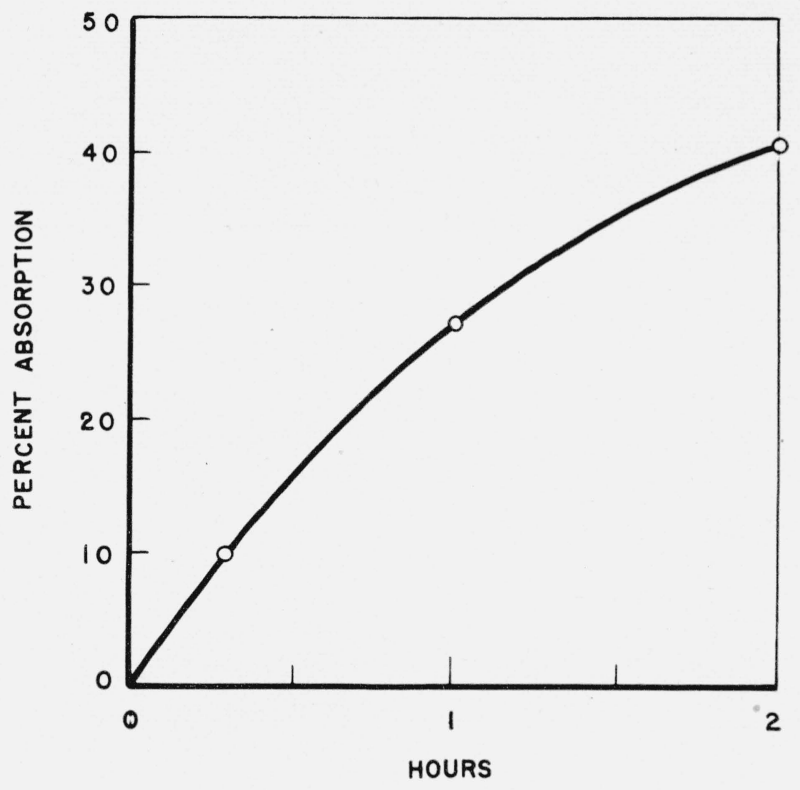

Figure 6.-Percentage absorption of radon by neoprene balloon during time in contact

Area of balloon $2.500 \mathrm{~cm}^{2}$.

radon lost under these conditions would be due not only to absorption but also to transmission through the neoprene.

\section{Additional Remarks}

A satisfactory arrangement when a sufficient number of chambers are available is to reserve some chambers for large samples and use the short resolving time circuit. The remaining chambers, if reserved for small samples, can be used either with the short or long resolving time circuit. A sample of $10^{-8}$ curie was used to calibrate one chamber connected to a short resolving time circuit and its value was found to fall on the same line of calibration points used for smaller rates of counting with this arrangement. This indicates that quantities of this order can be readily measured within the same limits of error as smaller samples.

The writer acknowledges the helpful criticism of L. F. Curtiss and the assistance in measurements by Christine Shaffer and Sarah Culpepper

Washington, July 28, 1947. 